\title{
Smarte Produkte
}

\section{Matthias Knoll · Susanne Strahringer}

Das Schlagwort „Digitalisierung“ ist allgegenwärtig. Praktisch jeder Lebens- und Arbeitsbereich wird heute mit zunehmender IT-Durchdringung bestehender Produkte in Verbindung gebracht. Und tatsächlich: Immer leistungsfähigere und kleinere Hardware-Komponenten ermöglichen in Verbindung mit innovativen Konzepten neuartige Produkte und Dienstleistungen. Ihnen allen gemeinsam ist zum einen die mehr oder weniger umfangreiche additive IT-Funktionalität. Zum anderen aber sind diese Produkte und Dienstleistungen durch ihnen zugrunde liegende neue Geschäftsmodelle gekennzeichnet. Hierzu trägt vielfach auch das Merkmal bei, dass physische Produkte in der Lage sind, untereinander oder mit ihrer Umwelt zu kommunizieren.

Diese neu hinzugekommenen Fähigkeiten machen das Produkt oder die Dienstleistung „smart“. Eine solche Anreicherung um digitalen Komponenten, die anpassungsfähig sind an Situationen, Umgebungen und Kunden und die mit anderen Produkten und Umgebungen interagieren, sind heute aus unserem Alltag kaum mehr wegzudenken und bilden die Elemente einer „Smart World“, die sich über alle Branchen, Unternehmen, aber auch Arbeits- und Lebensbereiche erstreckt. Vor praktisch jeden etablierten Begriff kann heute das Wort ,smart“ gesetzt werden: Smart Factory, Smart Energy, Smart Traffic, Smart Health, Smart Home, um nur einige zu nennen.

Doch was genau steckt hinter diesem Begriff „,smart“. Ein Blick ins Wörterbuch bietet eine Vielzahl von Übersetzungsalternativen. Intelligent, schlau und schick sind drei in diesem Kontext durchaus interessante Übersetzungen. Alle drei vermitteln

\footnotetext{
M. Knoll ( $\bowtie)$

Hochschule Darmstadt, Darmstadt, Deutschland

E-Mail: matthias.knoll@h-da.de

S. Strahringer

Technische Universität Dresden, Dresden, Deutschland

E-Mail: susanne.strahringer@tu-dresden.de
} 
die gleiche Botschaft: Das smarte Element unterstützt uns als Anwenderin und Anwender. Und es lässt ein bislang eher, ,gewöhnliches“ Produkt oder eine altbewährte Dienstleistung plötzlich wieder modern wirken, indem es Fähigkeiten hinzufügt, die wir uns vielleicht schon länger gewünscht haben, die bislang aber nicht realisierbar waren.

Entsprechend werden diese Aspekte auch in der Forschung betrachtet. Smarte Produkte sind dort seit dem Aufkommen von RFID-Technologien, Embedded und Cyberphysical Systems sowie dem Internet der Dinge Gegenstand der Vision allgegenwärtiger Informationsverarbeitung (Ubiquitous Computing). Im Kontext aktueller Digitalisierungsthemen rund um Industrie 4.0, Big Data, Künstliche Intelligenz und maschinelles Lernen, Cloud-/Edge Computing sowie Ökosystem- und Plattformstrategien haben sie eine neue Qualität erreicht. Die Nutzung von Daten durch sie und über sie sowie ihre Einbettung in übergeordnete Systeme ermöglichen eine andere Dimension von „Intelligenz und Autonomie“, die heute in einer bislang ungeahnten Form wertschöpfend genutzt wird und entsprechend neuartige Geschäftsmodelle realisieren hilft.

Einem Beitrag im Harvard Business Review aus dem Jahre 2014 von Porter und Heppelmann ist es zu verdanken, die Aufmerksamkeit auf diese einschneidenden Auswirkungen gelenkt zu haben. Zugleich haben es die Autoren verstanden, dem Begriff „smart, connected products“ die heute dominante Prägung zu geben, die auch diesem Heft zugrunde liegt.

Die vorliegende Ausgabe 327 der HMD greift aus diesem beinahe unendlichen Spektrum von Themen zur Entstehung, Entwicklung und Nutzung Smarter Produkte unterschiedliche Bereiche heraus. Nach einem Einführungsbeitrag, der die Entwicklungen und Möglichkeiten aufzeigt und strukturiert, folgen Beiträge aus unterschiedlichen Anwendungsbereichen beginnend mit eher methodischen Ansätzen hin zu konkreten Anwendungsbeispielen. Welche Optionen bieten sich, wenn die physikalischen Grenzen eines Produkte mit entsprechenden Zusatzfunktionalitäten aufgehoben werden? Beispielhafte Einsatzbereiche im Maschinenbau und in der Automobilindustrie werden aufgezeigt, ebenso wie in der Logistik. Selbstverständlich ist auch das Smart Home ein wichtiges Thema. Ein Beitrag untersucht, was smarte Komponenten im Rahmen ihres Einsatzes auslösen können. Haben smarte Produkte das Potential zur Stresserzeugung - statt dem eigentlich beabsichtigten Gegenteil? Und schließlich zeigt ein Beitrag, wie eine smarte Dienstleistung in der Lage ist, uns kontextbezogene Musikempfehlungen zu geben.

Die Beiträge in der Rubrik Spektrum widmen sich in dieser Ausgabe dem Disruptionspotential und typischen Implikationen der Blockchain-Technologie, der Frage der Führung im Zeitalter der Digitalisierung sowie der Entwicklung eines Vorgehensmodells zur Einführung mobiler IT-Lösungen. Sie runden damit über einen Blick in die aktuellen Nachbardisziplinen die Ausgabe ab.

Und schließlich werfen zwei Rezensionen einen Blick auf begleitende Literatur: So erschienen uns die Bücher ,Digitalisierung in Unternehmen - Von den theoretischen Ansätzen zur praktischen Umsetzung“ des Herausgeberteams Thomas Barton, Christian Müller und Christian Seel sowie ebenfalls der Herausgeberband „Service Engineering - Von Dienstleistungen zu digitalen Service-Systemen“"von Kyrill Mey- 
er, Stephan Klingner und Christian Zinke die ideale Ergänzung zu den Themen in dieser Schwerpunktausgabe.

Unser herzlicher Dank gilt allen Autorinnen und Autoren dieser Ausgabe, die ihr Wissen mit uns und Ihnen teilen und auf diesem Weg wertvolle Impulse für die Diskussion und Implementierung entsprechender Lösungen geben.

Wir wünschen Ihnen eine spannende Lektüre, aus der Sie viel Wissen mitnehmen können, und freuen uns über Ihr Lob, aber auch über Ihre Anregungen und Kritik.

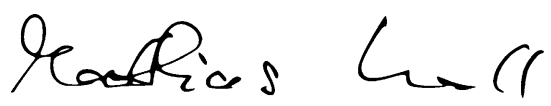

Matthias Knoll

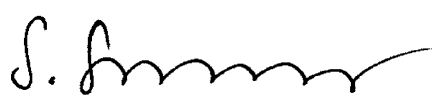

Susanne Strahringer 